\title{
AVALIAÇÃO DA TRANSFERÊNCIA DE CALOR EM MODELO DE COMBUSTÃO DE DUAS ZONAS PARA O ETANOL COMBUSTÍVEL.
}

\author{
J. L. S. FAGUNDEZ ${ }^{1}$ e N. P. G. SALAU \\ ${ }^{1}$ Universidade Federal de Santa Maria, Departamento de Engenharia Química \\ E-mail para contato: ${ }^{1}$ jeanluccasf@gmail.com
}

\begin{abstract}
RESUMO - Diversas correlações de transferência de calor da literatura podem ser utilizadas a fim de obter o coeficiente de transferência de calor dos gases de combustão em contato com as paredes do cilindro em um motor a combustão interna ciclo Otto. Para avaliar qual dessas correlações é capaz de melhor ajustar dados experimentais de pressão no interior do cilindro, foi feito um estudo comparativo entre a correlação de Eichelberg, que considera a convecção natural dos gases de combustão, com as correlações de Woschni, Hohenberg, Sitkei e Annand, que consideram a convecção forçada desses gases. As comparações foram realizadas utilizando o software Matlab® e considerando os ensaios de duas condições operacionais do motor operando com o etanol combustível: a) ponto de ignição fixo e b) ponto de ignição avançado para a obtenção do torque máximo do motor. Os resultados obtidos mostraram que a correlação de Hohenberg é a mais adequada para o tipo de motor e combustível utilizados.
\end{abstract}

\section{INTRODUÇÃO}

A produção e a utilização de fontes de energia renováveis de maneiras eficientes tornam-se pré-requisitos para a garantia de desenvolvimento econômico, segundo Tolmasquim et al. (2007). Assim, o etanol é visto como uma opção de energia renovável a ser explorada na utilização em motores de combustão interna. Parte dessa eficiência pode ser analisada com o estudo da transferência de calor que ocorre nesse tipo de motor.

Segundo Torregrosa et al.(2008), os efeitos da transferência de calor sobre o desempenho do motor e seu projeto mecânico conduzem a maioria das pesquisas relacionadas à transferência de calor do motor. Trabalhos experimentais e teóricos são realizados para caracterizar o comportamento térmico do motor com os objetivos de melhorar seu desempenho, reduzindo o consumo de combustível e as emissões, além de melhorar todo o gerenciamento térmico do veículo.

O tipo de análise realizada neste trabalho considera um modelo de duas zonas para a combustão. Modelos desse tipo podem representar uma ferramenta de simulação muito 
interessante, especialmente para motores com ignição por centelha (do inglês spark ignition, SI), devido ao tipo de combustão. A frente de chama separa a câmara em uma zona quente "queimada" e uma zona "não queimada" muito mais fria, de acordo com Lounici et al. (2010). O uso desses modelos implica em diferentes opções para a correlação usada para avaliar a transferência de calor, sendo as correlações de Woschni, Hohenberg e Annand as mais comuns.

Os testes foram realizados tanto com ponto de ignição avançado para MBT (do inglês Maximum Brake Torque) como para Ponto Fixo de ignição do motor.

\section{EQUACIONAMENTO TERMODINÂMICO DO MODELO}

A primeira lei da termodinâmica para um sistema aberto é representada na Equação 1:

$$
\dot{E}=\dot{Q}-\dot{W}+\sum_{j=1}^{2} \dot{m}_{\iota} h_{l}
$$

A taxa de transferência de energia no sistema, $\dot{E}$, é igual à diferença entre o calor gerado ou perdido em cada parte da superfície de controle, $\dot{Q}$, e o trabalho realizado pelo sistema, $\dot{W}$, sendo a unidade de medida em $\mathrm{J} / \mathrm{kg} \cdot \mathrm{s}$. O produto $\dot{m}_{l} \cdot h_{l}$ contabiliza o valor líquido entre a entrada e a saída de energia decorrente da alteração de estado do fluido de trabalho (Heywood, 1988), com a massa $\dot{m}_{l}[\mathrm{~kg} / \mathrm{s}]$ contabilizando a perda por blow-by e a entalpia $h_{l} \mathrm{em} \mathrm{J} / \mathrm{kg}$.

Para o modelo utilizado no presente trabalho, considerando o volume de controle como sendo o cilindro onde se dará o processo de combustão, o objetivo é definir a mudança de estado termodinâmico no decorrer do ciclo em função do ângulo de virabrequim, $\theta$. Assim, substituindo a energia e o trabalho e derivando a Equação 1 em função do ângulo do virabrequim, com energia interna $(u)$ em $\mathrm{J} / \mathrm{kg}$, pressão $(P)$ em Pa e volume $(V)$ em $\mathrm{m}^{3}$, obtém-se a Equação 2:

$$
m \frac{d u}{d \theta}+u \frac{d m}{d \theta}=\frac{d Q}{d \theta}-P \frac{d V}{d \theta}+\frac{d\left(\dot{m}_{l} h_{l}\right)}{d \theta}
$$

Como o modelo considera um motor no qual as válvulas estão fechadas, a variação de massa no cilindro decorre somente de perdas decorrentes de vazamentos nos anéis (Ferguson, 1986), sendo representada pela constante $\omega[1 / \mathrm{s}]$ de blow-by, como mostra a Equação 3:

$$
\frac{d\left(\dot{m_{l}} \dot{h_{l}}\right)}{d \theta}=-\frac{\dot{m}_{b} h_{b}}{\omega}
$$

Substituindo a Equação 3 na Equação 2 obtemos a forma geral da equação de energia para um volume de controle que acomode o conteúdo do cilindro:

$m \frac{d u}{d \theta}+u \frac{d m}{d \theta}=\frac{d Q}{d \theta}-P \frac{d V}{d \theta}-\frac{m_{b} h_{b}}{\omega}$ 
A variação de calor no sistema, representada na Equação 4, pode ser expressa em termos do calor transmitido pelas zonas "queimada" $\left(Q_{b}\right)$ e "não queimada" $\left(Q_{u}\right)$, como mostra a Equação 5:

$\frac{d Q}{d \theta}=\frac{-Q_{b}-Q_{u}}{\omega}$

Para expressar o calor perdido em termos da temperatura é necessária a introdução de um coeficiente global de troca térmica $h c$, que a partir da equação de Newton para transferência convectiva de calor, segundo Lounici et al. (2010), com a área $\left(A_{b}\right.$ e $\left.A_{u}\right)$ em $\mathrm{m}^{2}$ e o $h c$ em $\mathrm{W} / \mathrm{m}^{2} . \mathrm{K}$, gera as Equações 6 e 7 , de $Q_{b}$ e $Q_{u}$ :

$Q_{b}=h c A_{b}\left(T_{b}-T_{w}\right)$

$Q_{u}=h c A_{u}\left(T_{u}-T_{w}\right)$

Nestas equações, o coeficiente de troca térmica $(h c)$ deve ser avaliado para determinar as trocas térmicas que ocorrem em cada ciclo do motor. Para a obtenção desse coeficiente, serão usados submodelos apresentados a seguir.

\section{SUBMODELOS DE TRANSFERÊNCIA DE CALOR}

Durante a combustão, a temperatura dos gases queimados aumenta significativamente com um máximo que pode chegar a $2800 \mathrm{~K}$. A transferência de calor entre gases e as paredes da câmara é não-uniforme e transiente, tornando-se geralmente complexa para avaliar. Muitas aproximações podem ser usadas, dependendo do tipo de resultado requerido. Esses submodelos de transferência de calor, chamados comumente de correlações, podem ser classificados em duas categorias, de acordo com a origem da transferência de calor por convecção natural ou forçada.

Na tabela 1 estão presentes as variáveis usadas nas correlações de transferência de calor, suas definições e suas unidades de medida:

Tabela 1 - Parâmetros das correlações de transferência de calor

\begin{tabular}{|c|c|c|}
\hline Variável & Definição & Unidade de medida \\
\hline$P$ & Pressão no interior do cilindro & bar \\
\hline$P_{1}$ & Pressão na admissão & bar \\
\hline$P_{m o t}$ & Pressão de ensaio motored & bar \\
\hline$V$ & Volume no interior cilindro & $\mathrm{m}^{3}$ \\
\hline$V_{d}$ & Volume varrido pelo pistão & $\mathrm{K}$ \\
\hline$T$ & Temperatura no interior do cilindro & $\mathrm{K}$ \\
\hline$T_{1}$ & Temperatura na admissão & $\mathrm{K}$ \\
\hline$T_{g}$ & Temperatura da zona a ser avaliada & $\mathrm{K}$ \\
\hline$T_{w}$ & Temperatura da parede do cilindro & $\mathrm{m} / \mathrm{s}$ \\
\hline$v_{m p}$ & Velocidade média do pistão & $\mathrm{m}$ \\
\hline$B$ & Diâmetro do pistão & \\
\hline
\end{tabular}




\begin{tabular}{|c|c|c|}
\hline$A$ & Área da câmara de combustão & $\mathrm{m}^{2}$ \\
\hline$k_{g}$ & Coeficiente de troca térmica & $\mathrm{W} / \mathrm{m}^{2} / \mathrm{K}$ \\
\hline$R e$ & Número de Reynolds & Adimensional \\
\hline$C_{1}$ e $C_{2}$ & Constantes de Woschni e Hohenberg & Adimensionais \\
\hline$C_{3}$ & Constante de Woschni & $\mathrm{m} / \mathrm{s} / \mathrm{K}$ \\
\hline$a$ & Constante de Sitkei e Annand & Adimensional \\
\hline$b$ & Constante de Annand & $\mathrm{W} / \mathrm{m}^{2} / \mathrm{K}^{4}$ \\
\hline
\end{tabular}

\subsection{Convecção Natural}

O único submodelo que assume convecção natural para a transferência de calor dos gases de combustão que será considerado neste trabalho é o de Eichelberg. Esta correlação, embora faça parte de um grupo que acabou sendo abandonado em favor do uso de análise dimensional considerando convecção forçada, continua dando boas estimações para o cálculo de transferência térmica (Torregrosa et al., 2008). A correlação de Eichelberg pode ser vista na Equação 8, a seguir:

Correlação de Eichelberg (1939):

$h c=7,67 \times 10^{-6}\left(v_{m p}\right)^{\frac{1}{3}}\left(P T_{g}\right)^{\frac{1}{2}}$

\subsection{Convecção Forçada}

Devido à inadequação de supor convecção natural, o uso dessas correlações se aplica de maneira difícil aos motores. Dessa forma, alguns pesquisadores adotaram a suposição de convecção forçada. Essa suposição é mais realista devido aos movimentos do fluido na câmara serem consequência de ações mecânicas externas.

As correlações utilizadas dessa categoria são as de Woschni, Hohenberg, Sitkei e Annand, nas Equações 9, 10, 11 e 12, respectivamente:

Correlação de Woschni (1967):

$h c=C_{1}\left[B^{-0,2} P^{-0,8}\left(\left(C_{2} v_{m p}\right)+C_{3} \frac{V_{d} T_{1}}{P_{1} T_{1}}\left(P-P_{m o t}\right)\right)^{0,8} T^{-0,53}\right]$

Correlação de Hohenberg (1979):

$h c=C_{1} V^{-0,06} P^{0,8} T^{-0,4}\left(C_{2}-v_{m p}\right)^{0,8}$

Correlação de Sitkei (1972): 
$h c=2,36 \times 10^{-4}(1+a) \frac{\left(P v_{m p}\right)^{0,7} A^{0,3}}{T^{0,2}(4 V)^{0,3}}$

Correlação de Annand (1963):

$h c=a \frac{k_{g}}{B} R e^{0,7}+b \frac{\left(T_{g}{ }^{4}-T_{w}{ }^{4}\right)}{\left(T_{g}-T_{w}\right)}$

Os parâmetros que permitem a calibração de cada uma das correlações foram ajustados conforme estimações feitas utilizando o software Matlab® juntamente com o algoritmo de mínimos quadrados não lineares formulado na função do software 'Isqnonlin'.

Para integração numérica das equações diferenciais utilizou-se a função "ode45" do software Matlab®, que tem como integrador padrão o método de Dormand-Prince, uma das técnicas associadas ao método de Runge-Kutta (Dormand e Prince, 1980).

\section{CONFIGURAÇÃO EXPERIMENTAL}

Os parâmetros geométricos do motor utilizado, bem como a razão de compressão usada para a queima do etanol hidratado podem ser vistos na Tabela 2 :

Tabela 2 - Parâmetros geométricos e razão de compressão do motor de testes

\begin{tabular}{|c|c|}
\hline Diâmetro do Pistão $(\mathrm{m})$ & 0,09 \\
\hline Deslocamento do Pistão $(\mathrm{m})$ & 0,105 \\
\hline Comprimento da Biela $(\mathrm{m})$ & 0,16 \\
\hline Volume do Ponto Morto Inferior $\left(\mathrm{m}^{3}\right)$ & $7,236 \times 10^{-4}$ \\
\hline Razão de Compressão & 12 \\
\hline
\end{tabular}

Os ensaios com Ponto Fixo (PF) consideraram o maior avanço de ignição possível para as condições operacionais de ensaio antes de o motor começar a apresentar problemas, enquanto os ensaios de Maximum Brake Torque (MBT) foram feitos com o avanço de ignição que apresentasse a queima mais eficiente de combustível. As condições operacionais constam na Tabela 3:

Tabela 3 - Condições operacionais estabelecidas para ensaio PF e MBT

\begin{tabular}{|c|c|c|}
\hline Ensaio & PF & MBT \\
\hline Rotação $(\mathrm{rpm})$ & 1830 & 1805 \\
\hline$\Lambda$ & 1,07 & 1,08 \\
\hline Ponto de Ignição $\left({ }^{\circ} \mathrm{BTDC}\right)$ & $-6,5$ & $-6,5$ \\
\hline Duração da Combustão $\left({ }^{\circ}\right)$ & 58,8 & 53,1 \\
\hline Torque $($ N.m) & 33,8 & 33,3 \\
\hline Temperatura de Admissão $\left({ }^{\circ} \mathrm{C}\right)$ & 22 & 24 \\
\hline
\end{tabular}


A configuração do computador responsável pela simulação do modelo de duas zonas é mostrada na Tabela 4:

Tabela 4 - Configuração do computador responsável pela simulação

\begin{tabular}{|c|c|}
\hline Marca & Samsung Eletronics \\
\hline Processador & Intel ${ }^{\circledR}$ Core $^{\mathrm{TM}} 2$ Quad \\
\hline $\mathrm{CPU}$ & Q8200@2.33GHz \\
\hline Memória Instalada (RAM) & $2 \mathrm{~GB}$ \\
\hline Sistema Operacional & Windows 7 Profissional \\
\hline Tipo de Sistema & 32 bits \\
\hline
\end{tabular}

Para os dois tipos de ensaios, a temperatura da parede externa do cilindro e a constante de blow-by foram mantidas constantes e iguais a $420 \mathrm{~K} \mathrm{e} 0$.

\section{AVALIAÇÃO DOS RESULTADOS}

Os resultados para os ensaios de Ponto Fixo e Maximum Brake Torque serão mostrados em sequência.

\subsection{Ponto Fixo:}

As Figuras 1.a e 1.b mostram os resultados para o ensaio de Ponto Fixo, sendo a primeira para o ciclo total avaliado e a segunda apenas para os ângulos de duração da combustão, entre $6,5^{\circ}$ e $52,3^{\circ}$ :

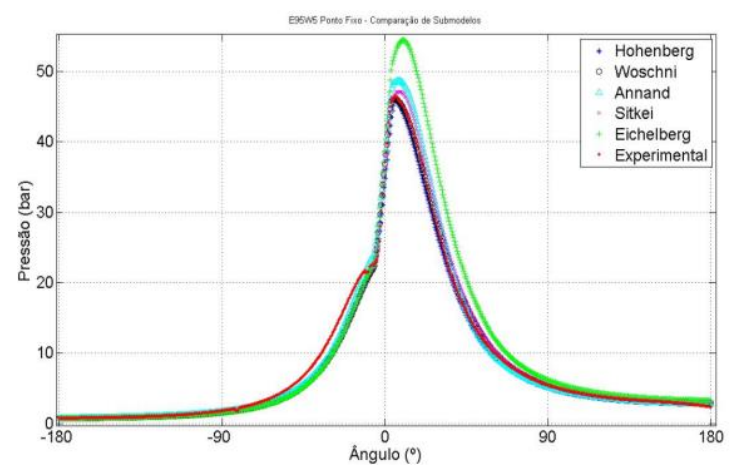

Figura 1.a. Ciclo total do cilindro.

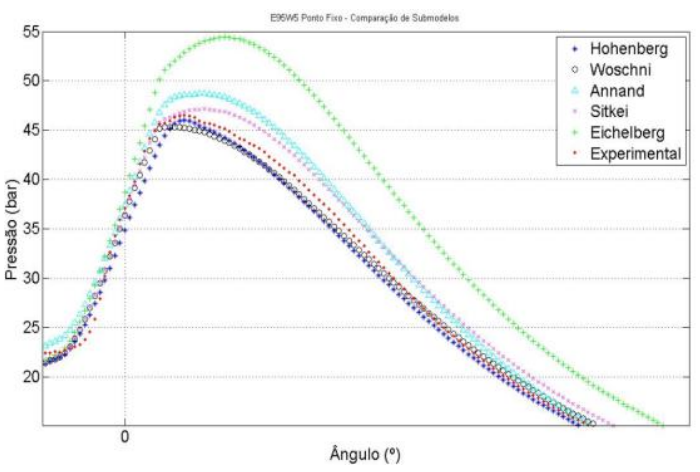

Figura 1.b. Fase de combustão. 


\subsection{Maximum Brake Torque:}

As Figuras 2.a e 2.b mostram os resultados para o ensaio de Maximum Brake Torque, sendo a primeira para o ciclo total avaliado e a segunda apenas para os ângulos de duração da combustão, entre $-6,5^{\circ}$ e $46,6^{\circ}$ :

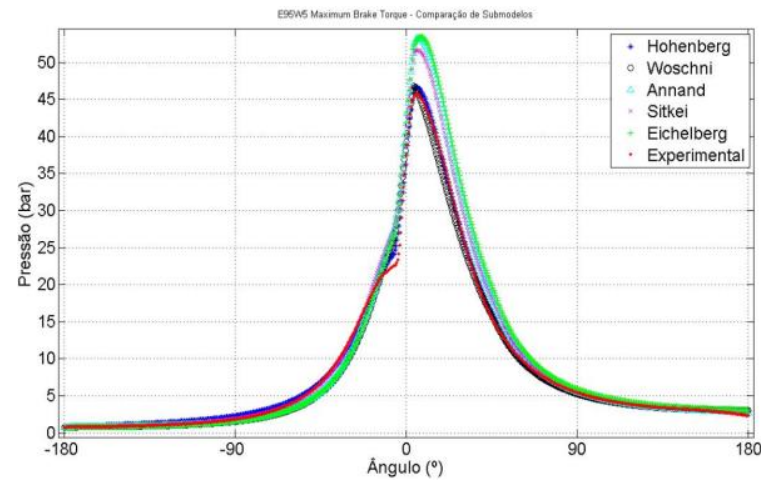

Figura 2.a. Ciclo total do cilindro.

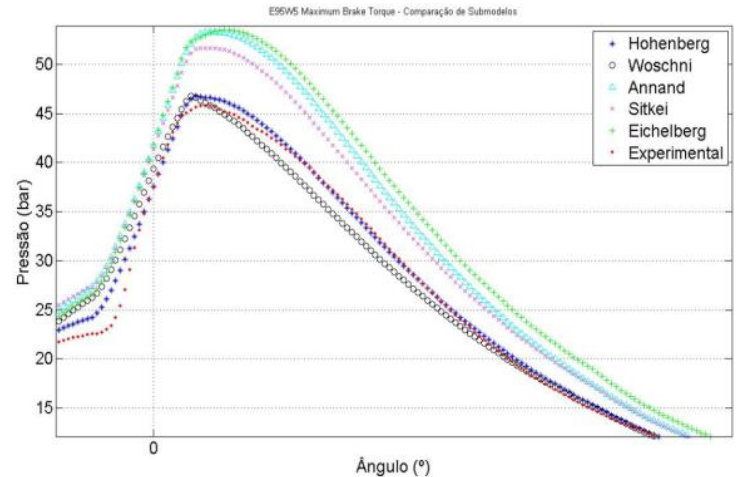

Figura 2.b. Fase de combustão.

É possível observar que o submodelo de transferência de calor de Hohenberg ajusta melhor os dados experimentais em ambos os ensaios. Para mostrar esse resultado com mais precisão foi calculada a correlação entre os valores gerados pelos submodelos e os dados experimentais, sendo o resultado mostrado através do coeficiente $R$ na Tabela 4:

Tabela 4 - Coeficientes de correlação $R$

\begin{tabular}{|c|c|c|}
\hline Ensaio & PF & MBT \\
\hline Hohenberg & 0,9979 & 0,9992 \\
\hline Woschni & 0,9975 & 0,9959 \\
\hline Annand & 0,9976 & 0,9966 \\
\hline Sitkei & 0,9958 & 0,9985 \\
\hline Eichelberg & 0,9888 & 0,9948 \\
\hline
\end{tabular}

O tempo de processamento para a simulação de Ponto Fixo com o submodelo de Hohenberg foi de 10,69 segundos, enquanto que a simulação de Maximum Brake Torque com o mesmo submodelo teve custo computacional de 12,50 segundos.

\section{CONCLUSÃO}

Como foi visto através dos gráficos obtidos para as simulações de Ponto Fixo e de Maximum Brake Torque, o submodelo de Hohenberg, responsável pelo melhor ajuste dos dados, é capaz de predizer com precisão os dados experimentais, além de ter um custo computacional muito pequeno em comparação ao uso de softwares mais robustos, como os CFDs. Além do 
submodelo de Hohenberg, merece menção também o submodelo de Woschni, que obteve bons resultados para os dois tipos de ensaios. Os demais submodelos conseguem prever de forma apurada as fases de compressão e expansão, mas a pressão na fase de combustão em geral é superestimada.

Considerados os erros inerentes às simplificações feitas por um modelo de duas zonas, a capacidade de predição deste tipo de modelo é muito interessante devido aos bons resultados gerados e ao custo computacional mínimo, já que qualquer computador atual é capaz de utilizá-lo e obter resposta quase imediata. Para conclusões mais abrangentes sobre a eficiência desses modelos e dos submodelos utilizados em conjunto, são necessários testes com diferentes configurações do motor utilizado nos ensaios e diferentes combustíveis.

\section{REFERÊNCIAS}

ANNAND, W. J. D.; Heat Transfer in the Cylinder of Reciprocating Internal Combustion Engines. Proc. Instn. Mech. Engrs. 177 (1963) 973-990.

DORMAND, J. R.; PRINCE, P. J. A Family of Embedded Runge-Kutta Formulae. Journal of Computational and Applied Mathematics, Volume 6, 1980, p. 19-26.

EICHELBERG, G.; Some New Investigations on Old Combustion Engine Problems, Engineering 148 (1939) 446-463.

FERGUSON, C. R.; Internal Combustion Engines, Applied Thermosciences. John Wiley \& Sons, 1986.

HEYWOOD, J. B. Internal Combustion Engine Fundamentals. McGraw-Hill, 1988.

HOHENBERG, G. F.; Advanced Approaches for Heat Transfer Calculations. SAE Paper No. $790825,1979$.

LOUNICI, M. S.; LOUBAR, K.; BALISTROU, M.; TAZEROUT, M. Investigation on heat transfer evaluation for a more efficient two-zone combustion model in the case of natural gas SI engines. Applied Thermal Engineering (2010), doi: 10.1016/j.applthermaleng.2010.09.012.

TOLMASQUIM, M. T.; GUERREIRO, A.; GORINI, R. Matriz Energética Brasileira - Uma Prospectiva. Revista Novos Estudos, v. 79, p. 47-79, 2007.

TORREGROSA, J.; OLMEDA, P. C.; ROMERO, C. A. Revising Engine Heat Transfer. Journal of Engineering Annals of Faculty of Engineering Hunedoara, TOME VI (2008), FASCICULE 3, (ISSN 1584-2673).

SITKEI, G., RAMANAIAH, G. V.; Rational Approach for Calculation of Heat Transfer in Diesel Engines. SAE Paper No. 720027, 1972. 
19 a 22 de outubro de 2014

Florianópolis/SC

WOSCHNI, G.; A Universally Applicable Equation for the instantaneous Heat Transfer Coefficient in the Internal Combustion Engine, SAE Paper No. 670931, 1967. 